\title{
The Engineering Properties of Wood-Wool Cement Walls
}

\author{
Huang Chung-Ho \\ Department of Civil Engineering, National Taipei University of Technology, Taipei City, Taiwan
}

\section{Email address:}

cdewsx.hch@gmail.com

\section{To cite this article:}

Huang Chung-Ho. The Engineering Properties of Wood-Wool Cement Walls. Science Discovery. Vol. 4, No. 5, 2016, pp. 330-335. doi: $10.11648 /$ j.sd.20160405.23

Received: October 7, 2016; Accepted: October 15, 2016; Published: November 5, 2016

\begin{abstract}
Taiwan is situated in the seismic belt and belongs to the subtropical climate. The abilities of heat insulation and seismic resistant of buildings in Taiwan were become more and more important. Fundamentally, it should develop a new materials suitable for use in hot and humid environment. This research aims to study the engineering properties of the wood-wool cement and compare with those of traditional building materials. The test results show that the unit weight of wood-wool cement boards is about $615 \mathrm{~kg} / \mathrm{m}^{3}$. The wood-wool cement boards collocate with the light-weight rigid frame and paste those construct the wood-wool cement light wall, its unit weight will increase to about $1580 \mathrm{~kg} / \mathrm{m}^{3}$; Compare with the unit weight $2300 \mathrm{~kg} / \mathrm{m}^{3}$ concrete, the unit weight of wood-wool cement boards and light wall will decrease $73 \%$ and $31 \%$, respectively. The wood-wool cement light wall is not only beneficial to convenience of construction but also increase the load-bearing capacity of buildings, future more, it is contribute to reduce the seismic force. The thermal conductivity of wood-wool cement boards and the wood-wool cement light wall are about $0.09 \mathrm{kcal} / \mathrm{m}-\mathrm{hr}-{ }^{\circ} \mathrm{C}$ and $0.58 \mathrm{kcal} / \mathrm{m}-\mathrm{hr}-{ }^{\circ} \mathrm{C}$, which compare with the test result, 1.25 $\mathrm{kcal} / \mathrm{m}-\mathrm{hr}-{ }^{\circ} \mathrm{C}$ of concrete with compressive strength $210 \mathrm{~kg} / \mathrm{cm}^{2}$ are obviously decrease. As bending strength and wind pressure test that we know, the wood-wool cement light wall is worse than reinforced concrete. But the mechanics of the wood-wool cement light wall corresponds to the wallboard strength requirement of Taiwan Building Code. From the economic analysis, the wood-wool cement boards possess higher economic benefits due to better energy efficiency. As we know from the above, it can be expected soon to use the wood-wool cement light wall in buildings.
\end{abstract}

Keywords: Wood-Wool Cement Boards, Thermal Conductivity, Wind Pressure Test

\section{木丝水泥板轻量墙之工程性质}

黄中和

土木工程学系, 国立台北科技大学, 台北市, 台湾

邮箱

cdewsx.hch@gmail.com

摘要：建筑物难以避免地将采用保温取凉及耐震性能较佳的材料, 为符合这个趋势, 根本上应从建筑材料上作相当的 改革。本研究结合轻量木丝与具强度水泥两种材料特色, 进行木丝水泥墙板的工程性质试验, 并将试验结果与被取代 之传统建材相比较, 从而探求此新材料于房屋建筑的适用性。试验结果显示, 木丝水泥板之单位重约为 $615 \mathrm{~kg} / \mathrm{m}^{3}$ 左右, 若搭配轻钢架及1公分厚之水泥浆修饰层建构而成之木丝水泥墙版, 其单位重提高至 $1580 \mathrm{~kg} / \mathrm{m}^{3}$ 左右, 相较于一般混凝 土的 $2300 \mathrm{~kg} / \mathrm{m}^{3}$ 单位重, 分别降低了有 $73 \%$ 及 $31 \%$, 不但有利于施工的便利及建筑物承载容量的增加, 对于地震力减 低也将有帮助。木丝水泥板及木丝水泥墙版之热传导系数实测值约为 $0.09 \mathrm{kcal} / \mathrm{m}-\mathrm{hr}-{ }^{\circ} \mathrm{C}$ 及 $0.58 \mathrm{kcal} / \mathrm{m}-\mathrm{hr}-{ }^{\circ} \mathrm{C}$, 相较 于抗压强度 $210 \mathrm{~kg} / \mathrm{cm}^{2}$ 混凝土之试验值 $1.25 \mathrm{kcal} / \mathrm{m}-\mathrm{hr}-{ }^{\circ} \mathrm{C}$ 也有明显的降低。在力学性质方面, 由抗弯强度及风压试验 可知, 木丝水泥墙版的力学性质虽然较劣于钢筋混凝土，但其性能已可符合建筑技术规则的墙版强度要求。 
关键词: 木丝水泥板, 热传导系数, 风压试验

\section{1. 引言}

1970年代的两次能源危机, 使得世界各先进国家深刻 体认到地球之能源是有限的, 也因而积极推动其节约能源 措施及建立管理机制, 俾能帮助于地球环境能源与资源之 永续发展 $[1,2]$ 。现今, 建筑耗用能源已成为一个国家总 耗能的重要因素, 以先进国家而言, 建筑耗能约占其总耗 能的 $30 \%$ $50 \%$, 故建筑节约能源已受到各国的普遍重视 $[3,4]$ 。对于总体能源之 $97 \%$ 需依赖进口的台湾而言, 节能 政策更是国家经济发展与生存竞争的命脉 $[5,6]$ 。目前国 内住商部分之耗能量约占台湾地区总耗能量的 $17 \%$, 尤其 甚者, 建筑空调尖峰耗电量更达全国夏季尖峰总用电量的 三分之一 [7]。若从建筑物的直接节能定义观之, 则包括 建筑物的空调、照明和动力 (电力) 等的节约。而台湾位处 亚热带气候区, 夏季日照充足, 使得气温居高不下, 大多 数建筑物必须依赖空调设备来降低室内温度, 以增加舒适 度。但台湾地区的电力系统负载数据显示 $[8,9]$, 耗电量 越来越高, 电力备转率则越来越低, 此情况已充分凸显改 善建筑外壳隔热性对建筑节约能源管理之重要性与迫切 性。若能以低传热性的木丝水泥板轻质墙体系统取代混凝 土, 应能有效地改善建筑外壳隔热性, 以使建筑物达到节 约能源的功效。

本研究旨于针对木丝水泥板与其组装后之墙版, 进行 系列的工程性质试验研究, 并将试验结果与传统建材比较
优劣, 从而探求该材料的特点, 并比较传统材料之经济效 益。

\section{2. 试验规划}

本研究共采用木丝水泥板及木丝水泥板轻质墙版等 两项材料进行各项试验。于木丝水泥板基本材料试验部分, 除了量测基本的容积比重及吸水后体积变化等物理试验 外, 为了求得该材料在隔热上之性能, 尚规划有热传导系 数试验。另外考虑木丝水泥板亦可直接取代木质建材应用 于兴建小木屋等建筑, 以发挥较木质建材更具耐潮湿的能 力, 本文亦规划木丝水泥板之吸脱湿性能试验, 以验证其 耐候性。木丝水泥板除了可应用于装饰材料外，结合支撑 架与水泥砂浆等材料亦可大幅提高其强度及工程性质, 以 应用于房屋外墙版及内隔间等构件, 发挥减轻结构体单位 重及隔热节能的特性。为了了解上述应用时, 木丝水泥板 轻质墙版之强度与工程特性, 本计划中尚规划有单位重试 验、热传导系数试验以及强度试验（抗压、抗弯及风压试 验) 等。

\section{3. 木丝水泥板工程性质试验结果与分析}

为了了解实地应用于土木工程时, 木丝水泥板之隔热 节能与工程特性, 于本研究中进行物理性质、力学性质及 而候性等相关试验, 所测得之试验结果如表 $1 \sim$ 表 3 所示, 将所得之试验结果分析与讨论如后。

表1 木丝水泥板物理性质试验结果与规范值。

\begin{tabular}{|c|c|c|c|c|c|c|c|c|c|}
\hline \multirow{2}{*}{$\begin{array}{l}\text { 试体 } \\
\text { 编号 }\end{array}$} & \multirow{2}{*}{$\begin{array}{c}\text { 厚度 } \\
(\mathrm{mm})\end{array}$} & \multicolumn{2}{|c|}{ 质量 $\left(\mathrm{kg} / \mathrm{m}^{2}\right)$} & \multicolumn{2}{|c|}{ 容积比重 } & \multirow{2}{*}{$\begin{array}{l}\text { 热传导系数 } \\
\left(\mathrm{kcal} / \mathrm{m} . \mathrm{hr} .{ }^{\circ} \mathrm{C}\right)\end{array}$} & \multicolumn{2}{|c|}{ 热阻 $\quad\left(\mathrm{m}^{2} . \mathrm{hr} .{ }^{\circ} \mathrm{C} / \mathrm{kcal}\right)$} & \multirow{2}{*}{$\begin{array}{l}\text { 长度 } \\
\text { 变化 }\end{array}$} \\
\hline & & 试验值 & 规范值 & 试验值 & 规范值 & & 试验值 & 规范值 & \\
\hline 1 & 43 & 26.9 & \multirow{4}{*}{20 以上 } & 0.63 & \multirow{4}{*}{0.5 以上 } & 0.0836 & 0.514 & \multirow{4}{*}{0.43 以上 } & $0.04 \%$ \\
\hline 2 & 43 & 28.5 & & 0.66 & & 0.0866 & 0.496 & & $0.04 \%$ \\
\hline 3 & 42 & 25.1 & & 0.6 & & 0.09 & 0.466 & & $0.03 \%$ \\
\hline 平均 & & 26.8 & & 0.63 & & 0.0868 & 0.492 & & $0.04 \%$ \\
\hline
\end{tabular}

表2 木丝水泥板吸脱湿性能试验结果。

\begin{tabular}{|c|c|c|c|c|c|c|c|c|c|c|}
\hline & \multirow[b]{2}{*}{ RH } & \multirow[b]{2}{*}{ 初始质量（g） } & \multicolumn{4}{|c|}{ 木丝水泥板 } & \multicolumn{4}{|c|}{ 针叶树原木 } \\
\hline \multirow{5}{*}{$\begin{array}{l}\text { 吸 } \\
\text { 湿 } \\
\text { 試 } \\
\text { 验 }\end{array}$} & & & 172 & 167.7 & 145.9 & 平均 & 190.1 & 191.4 & 169.2 & 平均 \\
\hline & $75 \%$ & $6 \mathrm{hr} \quad(\%)$ & 1.3 & 1.1 & 1.4 & 1.3 & 1.4 & 1.6 & 1.5 & 1.5 \\
\hline & $\downarrow$ & $12 \mathrm{hr} \quad(\%)$ & 1.9 & 1.7 & 2.1 & 1.9 & 2.4 & 2.6 & 2.5 & 2.5 \\
\hline & $\mathrm{RH}$ & $18 \mathrm{hr} \quad(\%)$ & 2.2 & 2 & 2.3 & 2.1 & 2.7 & 3.1 & 2. 8 & 2.9 \\
\hline & $90 \%$ & $24 \mathrm{hr} \quad(\%)$ & 2.4 & 2.3 & 2.5 & 2.4 & 3.1 & 3.6 & 3.3 & 3.3 \\
\hline \multirow{5}{*}{$\begin{array}{l}\text { 脱 } \\
\text { 湿 } \\
\text { 验 } \\
\text { }\end{array}$} & RH & 初始质量（g） & 176.2 & 171.8 & 149. 8 & & 196.5 & 198.9 & 174.9 & \\
\hline & $90 \%$ & $6 h r \quad \%)$ & -0.6 & -0.7 & -0.9 & -0.8 & -1.6 & -1.6 & -1.7 & -1.6 \\
\hline & $\downarrow$ & $12 \mathrm{hr} \quad(\%)$ & -1 & -0.9 & -1.3 & -0.8 & -1.9 & -1.8 & -2 & -1.6 \\
\hline & $\mathrm{RH}$ & $18 \mathrm{hr} \quad(\%)$ & -1 & -1 & -1.2 & -1.1 & -1.9 & -1.9 & -1.9 & -1.9 \\
\hline & $75 \%$ & $24 \mathrm{hr} \quad(\%)$ & -1 & -1 & -1.2 & -1.1 & -2 & -1.9 & -2 & -1.9 \\
\hline
\end{tabular}


表3 木丝水泥板强度性质试验结果。

\begin{tabular}{|c|c|c|c|c|c|c|c|c|c|c|c|}
\hline \multirow{3}{*}{$\begin{array}{l}\text { 试体 } \\
\text { 编号 }\end{array}$} & \multirow{3}{*}{$\begin{array}{l}\text { 抗压 } \\
\text { 强度 } \\
\left(\mathrm{kg} / \mathrm{cm}^{2}\right)\end{array}$} & \multicolumn{10}{|c|}{ 抗弯强度与挠度 } \\
\hline & & \multirow{2}{*}{ 厚度 (mm) } & \multicolumn{2}{|c|}{ 抗弯破坏载重 (kgf) } & \multicolumn{2}{|c|}{ 挠度 $(\mathrm{mm})$} & \multirow{2}{*}{$\begin{array}{c}\begin{array}{l}\text { 厚度 } \\
(\mathrm{mm})\end{array} \\
\text { ( }\end{array}$} & \multicolumn{2}{|c|}{ 抗弯破坏载重 (kgf) } & \multicolumn{2}{|c|}{ 挠度 $(\mathrm{mm})$} \\
\hline & & & 试验值 & 规范值 & 试验值 & 规范值 & & 试验值 & 规范值 & 试验值 & 规范值 \\
\hline 1 & 27.4 & & 104.0 & & 5.80 & & & 475.3 & & 2.90 & \\
\hline 2 & 25.5 & 25 & 99.9 & 80 & 6.22 & 8 & 50 & 495.5 & 250 & 2.77 & 5 \\
\hline 3 & 27.0 & & 96.4 & 以上 & 6.43 & 以下 & & 486.4 & 以上 & 2.76 & 以下 \\
\hline 平均 & 26.6 & 平均 & 100.1 & & 6.15 & & 平均 & 485.7 & & 2.81 & \\
\hline
\end{tabular}

\section{1. 物理性质试验}

本试验共采用三片厚度 $45 \mathrm{~mm}$ 木丝水泥板进行单位面 积质量与容积比重试验, 试验结果如表1所示, 测得之单 位面积质量分别为 $25.1 、 26.9$ 及 $28.5 \mathrm{~kg} / \mathrm{m}^{2}$, 而对应之容 积比重则为 $0.56 、 0.60 、 0.63$, 试验之平均值分别为 26.8 $\mathrm{kg} / \mathrm{m}^{2}$ 与 0.60 , 变异系数也仅有 $6 \%$, 均符合CNS 9456 规范 中, 厚 $45 \mathrm{~mm}$ 木丝水泥板单位面积质量大于 $20.0 \mathrm{~kg} / \mathrm{m}^{2}$ 与容 积比重大于 0.50 的规定, 显示木丝水泥板材质量稳定且有 良好的水泥与木丝混合比例。试体尺寸为 $25 \times 5 \times 4$ 公分木 丝水泥板测得之长度变化量介于 $0.031 \% \sim 0.043 \%$, 平均 值为 $0.0367 \%$, 相较于普通混凝土干燥收缩量 $0.04 \sim 0.1 \%$ $\left[10^{\sim} 12\right]\left(400 \sim 1000 * 10^{-6}\right)$, 木丝水泥板的长度变化相若于 混凝土的变化量, 显示木丝水泥板拥有良好的体积稳定性。

热传导系数试验是依照CNS 7332 之规范来量测, 将 欲量之试片, 使其达到绝干状态, 放入热传导仪温箱中进 行热传导系数量测, 测得三片厚度 $45 \mathrm{~mm}$ 木丝水泥板之热 传导系数分别为 $0.0836 、 0.0866$ 及 $0.0900 \mathrm{kcal} / \mathrm{m} . \mathrm{hr} .{ }^{\circ} \mathrm{C}$, 试验之平均值分别为 $0.0868 \mathrm{kcal} / \mathrm{m} . \mathrm{hr} .{ }^{\circ} \mathrm{C}$ 。量测试体的 厚度, 并将试体之厚度除以热传导系数可得该材料之热阻 值, 其计算值介于 $0.466 \sim 0.514 \mathrm{~m}^{2} . \mathrm{hr} .{ }^{\circ} \mathrm{C} / \mathrm{kcal}$ 之间, 平均值约为 $0.49 \mathrm{~m}^{2} . \mathrm{hr} .{ }^{\circ} \mathrm{C} / \mathrm{kcal}$, 均符合CNS 9456规范 中, 厚 $45 \mathrm{~mm}$ 木丝水泥板热阻值须大于 $0.43 \mathrm{~m}^{2} . \mathrm{hr} .{ }^{\circ} \mathrm{C} / \mathrm{kcal}$ 的规定; 相较于一般混凝土材料之热传导系数界于 1. $0^{\sim} 1.5 \mathrm{kcal} / \mathrm{m} . \mathrm{hr} .{ }^{\circ} \mathrm{C}[13,14]$, 显示木丝水泥板拥有良 好的节能隔热功效。

\section{2. 而候性质试验}

为了模拟木丝水泥板在干燥与潮湿气候中的质量变 化, 本文拟进行吸脱湿性能试验, 乃将木丝水泥板与针叶 数原木置于相对湿度变化于 $75 \% \sim 90 \%$, 温度为 $30^{\circ} \mathrm{C}$ 之环 境中, 观察不同时间的质量变化情形, 所得之结果如表 2 所示。试验结果显示, 相对湿度由 $75 \%$ 提高为 $90 \%$ （湿度 由干燥转为潮湿的环境下) 时, 木丝水泥板在 $6 、 12 、 18$ 、 24 小时时平均增加的质量分别为 $1.3 \% 、 1.9 \% 、 2.1 \%$ 及 $2.4 \%$, 而针叶树原木平均增加量则为 $1.5 \% 、 2.5 \% 、 2.9 \%$ 与 $3.3 \%$, 当相对湿度由 $90 \%$ 降低为 $75 \%$ (湿度由潮湿转 为干燥的环境下) 时, 木丝水泥板随时间延长其质量减少 量在 $0.8 \% \sim 1.1 \%$ 之间, 而针叶树原木的质量减少量在 $1.6 \% \sim 1.9 \%$ 间变化, 由此试验可知, 受潮湿度变化的气 候环境中, 木丝水泥板其质量稳定度均较针叶树原木来的 佳, 显示木丝水泥板已提升了针叶树原木的耐候性能。

\section{3. 力学性质试验}

表3为木丝水泥板强度试验结果, 由抗压试验可知木 丝水泥板之平均抗压强度约为 $33 \mathrm{~kg} / \mathrm{cm} 2$, 已可符合非承 重隔间构件之基本强度要求，但其抗压强度明显低于混凝 土强度, 因此并不建议该材料使用于主要受力的结构构件 之中。在抗弯强度测试方面, 共采用厚度 $25 \mathrm{~mm}$ 与 $50 \mathrm{~mm}$ 木丝水泥板各三片试样进行抗弯试验, 测得之平均抗弯破 坏载重分别为 $100.1 \mathrm{kgf}$ 及 $485.7 \mathrm{kgf}$, 木丝水泥板破坏载 重时中央挠曲值则分别为 $6.15 \mathrm{~mm}$ 及 $2.81 \mathrm{~mm}$, 不论载重或 挠曲值均远高于CNS 9456规范的要求, 显示试验木丝水泥 板材有一定的抗弯强度与抗变形能力。

\section{4. 木丝水泥轻质墙版工程性质试验结果与分析}

\section{1. 木丝水泥板轻质墙工法}

木丝水泥板轻质墙主要系镀锌槽铁架、木丝水泥板及 粉饰砂浆等材料的组合, 其组装的方式不同将导致轻质墙 工程性质的差异。本研究采用两种不同的工法进行木丝水 泥板轻质墙的组装, 工法一乃将一片厚度为 $50 \mathrm{~mm}$ 之木丝 水泥板直接以螺丝锁入直立的镀锌槽铁架中, 为了增强粉 刷层水泥浆与木丝水泥板的握裹能力, 于木丝水泥板安置 一片薄金属网, 最后于墙版两面施以厚度 $15 \mathrm{~mm}$ 之水泥浆, 即组装完成厚约 $90 \sim 100 \mathrm{~mm}$ 的木丝水泥板TYPE S轻质墙版, 墙版之结构剖面图1 (a) 所示。而工法二大致上相似于工法 一, 主要的差别在于工法二是采用两块 $19 \mathrm{~mm}$ 厚的木丝水 泥板，配合铁架，于两木丝水泥板之间保留有 $20 \sim 30 \mathrm{~mm}$ 左右的空隙, 加上两面 $15 \mathrm{~mm}$ 的粉刷层, 该工法组成的木 丝水泥板TYPE D轻质墙版厚度大约保持在 $100 \mathrm{~mm}$ 左右, 此 工法完成之示意图如图1(b) 所示。

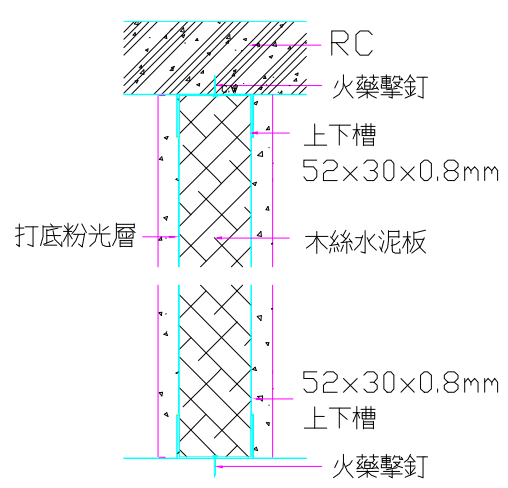

(a) 木丝水泥板TYPE S轻质墙版 


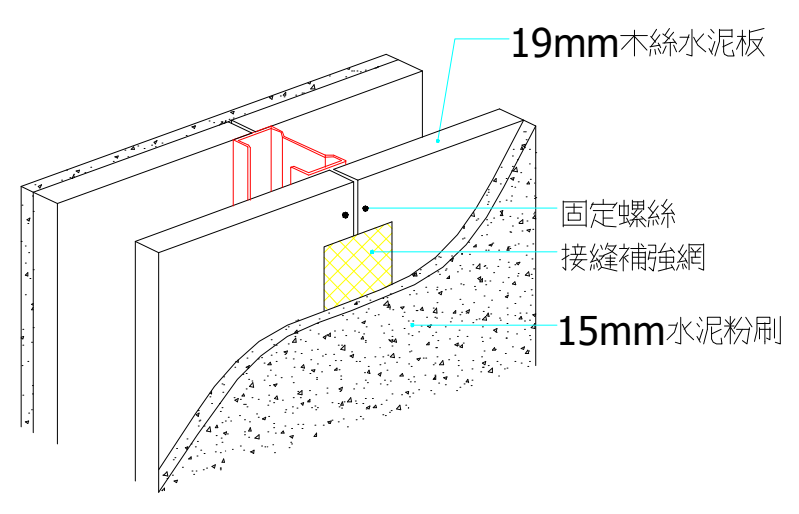

（b）木丝水泥板TYPE D轻质墙版

图1 木丝水泥板轻质墙版剖面图。

\section{2. 木丝水泥板轻质墙物理性质}

木丝水泥板轻质墙版基本物理性质试验包含单位重 与热传导系数试验, 试验结果陈列于表 4 之中。于单位重 试验中, 木丝水泥板TYPE S与TYPE D轻质墙版之单位重分 别为 $1455 \mathrm{~kg} / \mathrm{m} 3$ 及 $980 \mathrm{~kg} / \mathrm{m} 3$, 木丝水泥板轻质墙单位重 仅占一般RC墙单位重（ $2300 \mathrm{~kg} / \mathrm{m} 3 ）$ 的 $43 \% \sim 63 \%$, 成功 地达到使用木丝水泥板来降低墙体单位重的目的。在节能 隔热性能评估方面, 木丝水泥板TYPE S与TYPE D轻质墙版 之平均热传导系数分别为 $0.558 \mathrm{kcal} / \mathrm{m} . \mathrm{hr} .{ }^{\circ} \mathrm{C}$ 及 0.205 $\mathrm{kcal} / \mathrm{m} . \mathrm{hr} .{ }^{\circ} \mathrm{C}$, 经计算可得其热阻值分别为 $0.183 \mathrm{~m} 2 . \mathrm{hr} .{ }^{\circ} \mathrm{C}$ / kcal与 $0.502 \mathrm{~m} 2 . \mathrm{hr} .{ }^{\circ} \mathrm{C} / \mathrm{kcal}$ 。将木丝水泥泥轻质墙之 热传导系数与普通混凝土（热传导系数约介于 1.0 $\mathrm{kcal} / \mathrm{m} . \mathrm{hr} .{ }^{\circ} \mathrm{C} \sim 1.5 \mathrm{kcal} / \mathrm{m} . \mathrm{hr} .{ }^{\circ} \mathrm{C}$ 间）相比较，木丝水泥 板轻质墙热传导系数已可降低约 $60 \sim 85 \%$, 显示其节能隔 热性能要优于混凝土墙。

表4 木丝水泥板轻质墙版物理性质试验结果。

\begin{tabular}{lllllll}
\hline $\begin{array}{l}\text { 试体 } \\
\text { 编号 }\end{array}$ & $\begin{array}{l}\text { 长度 } \\
(\mathrm{mm})\end{array}$ & $\begin{array}{c}\text { 宽度 } \\
(\mathrm{mm})\end{array}$ & $\begin{array}{l}\text { 厚度 } \\
(\mathrm{mm})\end{array}$ & $\begin{array}{l}\text { 密度 } \\
\left(\mathrm{kg} / \mathrm{m}^{3}\right)\end{array}$ & $\begin{array}{l}\text { 热传导系数 } \\
\left(\mathrm{kcal} / \mathrm{m} . \mathrm{hr} .{ }^{\circ} \mathrm{C}\right)\end{array}$ & $\begin{array}{c}\text { 热阻 } \\
\left(\mathrm{m}^{2} . \mathrm{hr} .{ }^{\circ} \mathrm{C} / \mathrm{kcal}\right)\end{array}$ \\
\hline TYPE S1 & 200 & 200 & 103 & 1473 & 0.538 & 0.189 \\
TYPE S2 & 200 & 200 & 103 & 1452 & 0.587 & 0.174 \\
TYPE S3 & 200 & 200 & 103 & 1440 & 0.549 & 0.186 \\
平均 & 200 & 200 & 103 & 1455 & 0.558 & 0.183 \\
TYPE D1 & 200 & 200 & 103 & 984 & 0.195 & 0.528 \\
TYPE D2 & 200 & 200 & 103 & 971 & 0.201 & 0.513 \\
TYPE D3 & 200 & 200 & 102 & 985 & 0.218 & 0.467 \\
平均 & 200 & 200 & 103 & 980 & 0.205 & 0.502 \\
\hline
\end{tabular}

比较两不同工法组装的木丝水泥板轻质墙物理性质, 可发现TYPE D之单位重约为TYPE S者 $67 \%$, 而热传导系数 更仅占TYPE S的37\%, 主要原因乃为TYPE D墙版之中保留 了 $2 \sim 3$ 公分之空隙, 使得其物理性质相较于TYPE S 有明显 的提升。

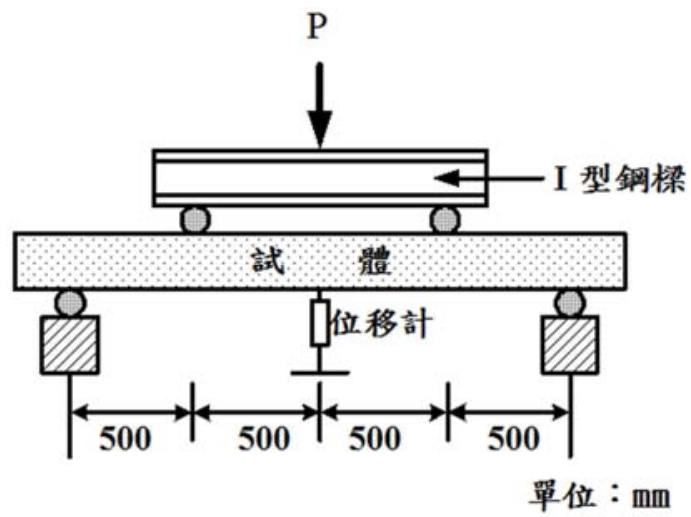

图2 木丝水泥板轻质墙抗弯强度示意图。

\section{3. 木丝水泥板轻质墙力学性质}

\section{A. 抗弯试验}

木丝水泥板轻质墙版抗弯试验装置如图2所示, 使用2 线载重装置, 加载跨度固定为 $200 \mathrm{~cm}$, 并于跨度中央放置 一位移计 (精度 $0.02 \mathrm{~mm}$ ) 量测中央变形量, 依照CNS 8081 建筑用组件性能检验法6.9节之规定进行试验, 加载平均
速率为 $2 \times 10-2 \mathrm{~mm} / \mathrm{sec}$, 记录破坏之加载荷重与中央挠度, 并依规范计算相当单位面积之弯矩破坏载重 $(\mathrm{Pm})$, 其计 算方式为 $\mathrm{Pm}=$ 最大载重/（跨度 $\times$ 试体宽度）。

表5 木丝水泥板轻质墙版抗弯强度试验结果。

\begin{tabular}{lllll}
\hline 试体编号 & $\begin{array}{l}\text { 试体尺寸 } \\
(\mathrm{cm})\end{array}$ & 破坏载重 $(\mathrm{kgf})$ & 中央挠度 $(\mathrm{mm})$ & $\mathrm{Pm}\left(\mathrm{kgf} / \mathrm{m}^{2}\right)$ \\
\hline TYPE S & $217 * 100 * 10$ & 672 & 40.16 & 336 \\
TYPE D & $230 * 100 * 10$ & 792 & 68.93 & 396 \\
\hline
\end{tabular}

依上述试验方法与计算可得表 5 试验结果。由木丝水 泥板轻质墙版之弯矩试验可得其弯矩破坏载重, TYPE S 木丝水泥板轻质墙版抗弯破坏载重为 $672 \mathrm{kgf}$, TYPE D则 为792 kgf, 计算其相当单位面积之弯矩破坏载重 $(\mathrm{Pm})$ 分别为 $336 \mathrm{kgf} / \mathrm{m} 2$ 及 $396 \mathrm{kgf} / \mathrm{m} 2$, 而中央挠曲变形可高达 约 $4.0 \mathrm{~cm} \sim 6.8 \mathrm{~cm}$ 。试验结果显示, 其抗弯强度高于砖墙, 可适用于非承重墙之强度要求, 且木丝水泥板轻质墙版拥 有较高可变形能力, 换言之其延展性能亦比普通砖墙来的 佳, 其主要原因是木丝水泥板轻质墙中的轻钢架（骨架） 改善了木丝水泥板挠曲行为, 使得其整体的墙版有较高的 强度与较佳的延展性。另外, 比较两不同类型的木丝水泥 板轻质墙抗压强度可知, 虽然TYPE D墙版拥有较多空隙而 使其单位重降低, 但由于TYPE D墙版钢架排列于跨度垂直 方向, 与TYPE S的水平方向有所不同, 导致TYPE D抗弯强 度优于TYPE S之木丝水泥板轻质墙版。

B. 风压试验 
本试验主要考虑因风力产生之均布荷重 (风压力) 对 木丝水泥板轻质墙版的影响, 风压力大小的选定则依照建 筑技术规则第三十三条规则, 选定条件为全台湾岛内最大 风力等级 (200级), 建筑物高度限定为 150 公尺以下, 其 规定之风压力为 $300 \mathrm{kgf} / \mathrm{m}^{2}$ 。为模拟风压产生之均布荷重, 拟以水压替代相同大小之风压。将试体置于跨度为 $200 \mathrm{~cm}$ 的支承上, 加载面积为 $90 * 200 \mathrm{~cm}$, 将水压缓缓加载于墙 版之上, 压应力每增加 $50 \mathrm{kgf} / \mathrm{m}^{2}$ （水深增加约 $5 \mathrm{~cm}$ ), 记录墙版中央挠度与观察墙版是否有裂缝或破坏产生, 一
直到压应力达 $300 \mathrm{kgf} / \mathrm{m}^{2}$ (水深达 $30 \mathrm{~cm}$ ), 停止加压并 维持水压 30 分钟, 随之进行卸除。

表6为木丝水泥板轻质墙版风压试验结果, 试验结果 显示, 不论TYPE S或TYPE D墙版在压应力达 $300 \mathrm{kgf} / \mathrm{m}^{2}$, 并维持30分钟, 墙版均无裂缝或破坏产生, 且卸除后墙版 的挠曲变形恢复原加载前之变形, 说明水压力仍在弹性范 围内。综合上述分析讨论, 木丝水泥板轻质墙版之风压力 可符合建筑技术规则第三十三条, 建筑物高度限定为 150 公尺以下, 200 级风压力的规定。

表6 木丝水泥板轻质墙版风压试验结果。

\begin{tabular}{|c|c|c|c|c|c|c|}
\hline \multirow{2}{*}{ 载重阶段 } & \multirow{2}{*}{$\begin{array}{l}\text { 水深 } \\
(\mathrm{cm})\end{array}$} & \multirow{2}{*}{$\begin{array}{l}\text { 水压 } \\
\left(\mathrm{kgf} / \mathrm{m}^{2}\right)\end{array}$} & \multicolumn{2}{|l|}{ TYPE S } & \multicolumn{2}{|l|}{ TYPE D } \\
\hline & & & 中央挠度 & 试体状况 & 中央挠度 & 试体状况 \\
\hline \multirow{8}{*}{ 加载 } & 0 & 0 & 0.00 & 墙 & 0.00 & 墙 \\
\hline & 5 & 50 & 0.30 & 版 & 0.08 & 版 \\
\hline & 10 & 100 & 0.50 & 无 & 0.16 & 无 \\
\hline & 15 & 150 & 0.66 & 缝 & 0.27 & $\begin{array}{l}\text { 衣 } \\
\text { 琏 }\end{array}$ \\
\hline & 20 & 200 & 0.89 & 或 & 0.36 & 或 \\
\hline & 25 & 250 & 1.11 & 破 & 0.44 & 破 \\
\hline & 30 & 300 & 1. 29 & $\begin{array}{l}\text { 尓 } \\
\text { 产 }\end{array}$ & 0.52 & 乲 \\
\hline & 33 & 330 & 1. 42 & 生 & 0.56 & 生 \\
\hline \multirow{7}{*}{ 卸除 } & 30 & 300 & 1.28 & & 0.49 & \\
\hline & 25 & 250 & 1.10 & & 0.41 & \\
\hline & 20 & 200 & 0.87 & & 0.32 & \\
\hline & 15 & 150 & 0.63 & & 0.24 & \\
\hline & 10 & 100 & 0.48 & & 0.12 & \\
\hline & 5 & 50 & 0.28 & & 0.06 & \\
\hline & 0 & 0 & 0.00 & & 0.00 & \\
\hline
\end{tabular}

\section{5. 结论}

本研究已完成「木丝水泥板」与「木丝水泥板轻质墙 版」之工程性质试验, 依照试验结果与分析提出下列综合 的结论与建议。

(1) 木丝水泥板之单位面积质量为 $26.8 \mathrm{~kg} / \mathrm{m}^{2}$, 所对应 之容积比重为 0.63 , 热阻值为 $0.492 \mathrm{~m} 2 . \mathrm{hr} .{ }^{\circ} \mathrm{C} /$ $\mathrm{kcal}, 25 \mathrm{~mm}$ 与 $50 \mathrm{~mm}$ 厚之木丝水泥板其抗弯破坏 载重分别为 $100 \mathrm{kgf}$ 及 $485 \mathrm{kgf}$, 上述木丝水泥板 质量测试皆符合规范的质量要求。

(2) 于 $30^{\circ} \mathrm{C}$ 恒温下, 相对湿度由 $75 \sim 90 \%$ 变化条件下, 木丝水泥板吸水后的平均长度变化为 $0.0367 \%$, 而质量变化量也仅有 $1.0 \%$ ～ $2.4 \%$ 变化，显示其 拥有良好的体积稳定性与耐候性，应可将木丝水 泥板材直接应用于游泳池、三温暖、储藏室、地 下室潮湿空间及小木屋等建筑外墙或内隔间构件。

(3) 木丝水泥板轻质墙版之工程性质受其工法不同而 有所影响, 在物理性质方面, 工法一（TYPE S） 与工法二（TYPE D ）墙版之单位重分别为 1455 $\mathrm{kg} / \mathrm{m} 3$ 及 $980 \mathrm{~kg} / \mathrm{m} 3$, 热阻分别为 $0.183 \mathrm{~m} 2 . \mathrm{hr} .{ }^{\circ} \mathrm{C} /$ $\mathrm{kcal}$ 与 $0.502 \mathrm{~m} 2 . \mathrm{hr} .{ }^{\circ} \mathrm{C} / \mathrm{kcal}$, 主要是因为TYPE D 墙版之中保留了 $2 \sim 3$ 公分之空隙, 使得其物理性 质相较于TYPE S 有明显的提升。

(4) 由木丝水泥板轻质墙版之弯矩试验可得其弯矩破 坏载重, TYPE S木丝水泥板轻质墙版抗弯破坏载 重为 $672 \mathrm{kgf}$, TYPE D则为792 kgf。虽然TYPE D
墙版拥有较多空隙，但其铁架排列于墙版之跨度 垂直方向, 与TYPE S 的水平方向有所不同, 因此 TYPE D抗弯强度优于TYPE S之木丝水泥板轻质墙 版。风压试验方面, 不论TYPE S或TYPE D墙版在 压应力达 $300 \mathrm{kgf} / \mathrm{m}^{2}$, 并维持 30 分钟, 墙版均无裂 缝或破坏产生, 可符合建筑技术规则第三十三条, 建筑物高度限定为 150 公尺以下, 200 级风压力的 规定。

(5)木丝水泥板轻质墙版相较于混凝土墙之单位重 (约 $2300 \mathrm{~kg} / \mathrm{m}^{3}$ ) 可降低37 57\%, 而一般之混凝 土热传导系数约为 $1.0 \sim 1.5 \mathrm{kcal} / \mathrm{m} . \mathrm{hr} .{ }^{\circ} \mathrm{C}$, 在隔 热能力方面也可降低约 $65 \sim 85 \%$ 。由此可知, 若 以木丝水泥板轻质墙版取代混凝土墙, 不但可降 低建筑物的静载重而提高建筑物的耐震性能, 也 可有效阻隔热能之传递, 使建筑物能源消耗大幅 降低，提升建筑物节约能源的功效。

(6) 由抗压试验可知木丝水泥板之抗压强度明显低于 混凝土强度, 因此并不建议该材料使用于主要受 力的结构构件之中。

\section{参考文献}

[1]台湾内政部建筑研究所「建筑物耗能总量调查之研究」2001 年版P. 11-16

[2] 台湾内政部建筑研究所「绿建筑解说与评估手册」2001年 版P. 3-31 
[3] Lam, J. C. and Hui, S. C. M. , "A review of building energy standards and implications for Hong Kong”, Building Research and Information, 24 (3): 131-140, 1996.

[4] The BOCA National Energy Conservation Code/ 1993, Seventh Edition, Building Officials \& Code Administrators International, Inc., 1993.

［5］张世典、陈锦赐、蔡必超、余凌昌、林伟志，「建筑节约 能源综合指标之探讨」, 中华民国建筑学会第十三届建筑 研究成果发表会论文集, 第663-668页 (2001)。

[6] 颜聪, 「房屋建材之隔热性工学」, 兴大土木第十期, 第 6-11页 (1983)。

［7］台湾内政部营建署「营建知讯」月刊2001年11月号「建筑 物用电量状况调查」，第23-27页

[8］台湾电力公司工程月刊 648 期「台湾电力需求与供给」, 第 73页。

[9] 台湾电力公司工程月刊 653 期 $「$ 电力尖峰负载及平均负载成 长比较」，第87页。

[10] Lee, E., Park, S., Kim, Y., Drying shrinkage cracking of concrete using dune sand and crushed sand,
Construction and Building Materials, Vol. 126, pp. 517-526. (2016)

[11] Parsekian, G. A., Barbosa, K. C., Camacho, J. S., Felipe, A.S, Experimental shrinkage testing on concrete masonry units and walls with different curing conditions, Masonry International 28 (3), pp. 71-80. (2016)

[12] Zhang, T., Liang, X. a, Li, C., Lorin, M., Li, Y., Vandeperre, L. J., Cheeseman, C. R., Control of drying shrinkage in magnesium silicate hydrate $\left(\mathrm{m}^{-} \mathrm{s}^{-} \mathrm{h}\right)$ gel mortars Cement and Concrete Research, Vol. 88, pp. 36-42. (2016)

[13] Li, S., Jones, B., Thorpe, R., Davis, M., An investigation into the thermal conductivity of hydrating sprayed concrete, Construction and Building Materials, 124, pp. 363-372. (2016)

[14] Real, S., Bogas, J. A., Da Glória Gomes, M. , Ferrer, B., Thermal conductivity of structural lightweight aggregate concrete, Magazine of Concrete Research, 68 (15), pp. 798-808. (2016) 